\title{
Output-Capacitance Hysteresis Losses of Field-Effect Transistors
}

\author{
Nirmana Perera, Armin Jafari, Luca Nela, Georgios Kampitsis, Mohammad Samizadeh Nikoo and Elison Matioli \\ Power and Wide-Band-Gap Electronics Research Laboratory (POWERlab) \\ École Polytechnique Fédérale de Lausanne (EPFL) \\ Lausanne, Switzerland \\ nirmana.perera@epfl.ch and elison.matioli@epfl.ch
}

\begin{abstract}
Resonant-type power converters are supposed to generate zero switching losses during soft-switching operation. In recent research, unexpected switching losses were reported in these converters, which were attributed to a large-signal hysteresis observed in the output-charge versus voltage $(Q V)$ characteristics of the power device. Since these converters subject the power semiconductor switch to different levels of peak voltages based on design requirements, the dependence of hysteresis losses on the voltage range is important. In this work, planar$\mathrm{Si}, \mathrm{Si}$ super-junction, $\mathrm{SiC}$ and $\mathrm{GaN}$ power device structures are investigated, and categorized based on their $Q V$ patterns. We report that for different device technologies, the large-signal hysteresis patterns of output charge show diverse dependencies on the excitation voltage amplitude. The frequency dependence of the hysteresis losses is also analysed and discussed. The presented results provide important insights in identifying the root causes for output-charge hysteresis, and may help to improve device spice models to properly account for soft-switching losses.
\end{abstract}

Index Terms - $C_{\text {oss }}$, hysteresis loss, output capacitance, SawyerTower, soft-switching loss, wide-band-gap (WBG) devices.

\section{INTRODUCTION}

Resonant-type power converters are an attractive solution to achieving large power densities at high frequencies due to their soft-switching behaviour [1]-[3]. Their applications include, among others, computer power supplies [4], radio-frequency (RF) power amplifiers in communication systems [5]-[7], and wireless power transfer systems [8]. During a single switching cycle in these converters, the output capacitance, $C_{\mathrm{o}}$, of the switching device is charged and discharged in a resonant manner dictated by the specificities of the topology, as means of achieving soft-switching conditions [1]. Since the device is in OFF state during the charging-discharging process of $C_{\mathrm{o}}$, this ideally yields zero losses [2], [9]—-see Figs. 1(a) and 1(b) - in comparison to hard-switching conditions [10].

However, it has been reported in recent research that, transition losses still exist due to dynamic charging and discharging of the power device's output capacitance under soft-switching conditions [2], [11]-[15]. The corresponding energy loss is attributed to a hysteresis loss [9], which is observed in large-signal charge versus voltage $(Q V)$ curves as shown in Fig. 1(c). Numerous works have reported such losses pertaining to different device structures: in Si Super-

This work was supported by the Swiss Office of Energy (SFOE) under Grant SI/501887-01 (MEPCO).

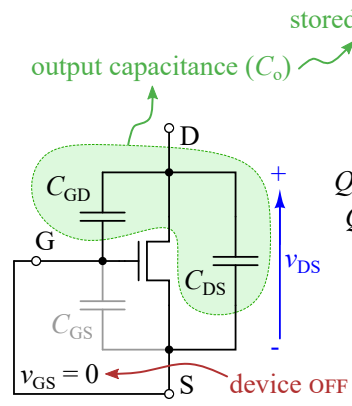

(a) ored charge $=Q_{0}$

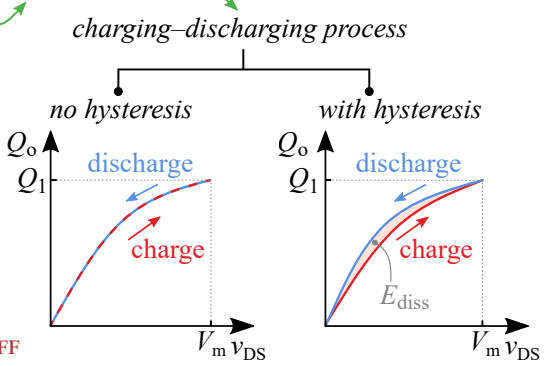

(b)

(c)

Fig. 1. (a) In resonant-type converters, the charging-discharging process of the output capacitance $\left(C_{\mathrm{o}}=C_{\mathrm{GD}}+C_{\mathrm{DS}}\right)$ of a transistor occurs while it is in OFF state, i.e., $v_{\mathrm{GS}}=0 \mathrm{~V}$. The related variation of the accumulated charge $\left(Q_{\mathrm{o}}\right)$ versus device drain-source voltage, $v_{\mathrm{DS}}$, is represented by a $Q V$ curve. Two cases are possible: (b) without hysteresis; and (c) with hysteresis, where different charging (red line) and discharging (blue line) paths result in a hysteresis energy loss, $E_{\text {diss }}$, which is specified for a given maximum voltage $V_{\mathrm{m}}$ of $v_{\mathrm{DS}}$.

Junction (Si-SJ) transistors [13], [16], [17]; and wide-bandgap (WBG) SiC transistors [15], [18] and GaN high-electronmobility transistors (HEMTs) [2], [14], [15], [19], [20].

The hysteresis energy loss in $C_{\mathrm{o}}$ is a function of the maximum (or peak) voltage, $V_{\mathrm{m}}$, across the device's drainsource terminals, and is expressed as

$$
E_{\mathrm{diss}}=\int_{0}^{Q_{1}} v_{\mathrm{DS}} d Q-\int_{Q_{1}}^{0} v_{\mathrm{DS}} d Q
$$

where $Q_{1}=\left.Q_{\mathrm{o}}\right|_{v_{\mathrm{DS}}=V_{\mathrm{m}}}$. This energy loss adds an extra constraint on deciding the maximum voltage across a switching device for a given high- or very-high-frequency (HF or VHF) application. This is especially important in resonant converters, for example, in the classical class-E inverter where the device voltage stress could be quite high [5]. Even for converters with low voltage stresses, such as the class- $\phi_{2}$ inverter [6], the circuit components are selected and tuned to achieve a given $V_{\mathrm{m}}$ value. Prior knowledge on the dependence of hysteresis energy losses with $V_{\mathrm{m}}$, for the available devices, would allow to select the most suitable operational voltage range for the circuit. In light of this, the quantitative variation of the $E_{\text {diss }}$ value with $V_{\mathrm{m}}$ has been published for some commercial devices [2], [15], [18]. Although this provides means of selecting a suitable device with lower losses, the 
understanding of how $Q V$ patterns vary with $V_{\mathrm{m}}$ for different device technologies is important in identifying the root cause for $E_{\text {diss }}$, and thus improving the device performance. This also aids in developing more realistic spice models to account for hysteresis losses.

In this work, four prominent field-effect transistor (FET) technologies are examined to observe how their $Q V$ patterns change with $V_{\mathrm{m}}$. This allows the identification of voltage ranges upon where the hysteresis patterns emerge. The frequency dependencies are also studied. An overview on the $Q V$ hysteresis patterns between different device structures is provided, which is lacking in the literature. The paper is structured as follows. Section II describes the measurement process and introduces the basics of output capacitance hysteresis. Section III and Section IV investigate the dependence of $Q V$ patterns on excitation voltage and frequency, respectively. Section V provides a survey on the origin of $E_{\text {diss }}$ losses in different device technologies and categorizes $Q V$ hysteresis patterns. Section VI concludes the paper.

\section{Measurement of Output-Capacitance HYSTERESIS}

This study concerns soft-switching losses in the frequency range of $10 \mathrm{kHz}$ to $1 \mathrm{MHz}$. The test circuit operates such that the excitation voltage across the device drain-source terminals is of sinusoidal nature, while the gate-source terminals are shorted, i.e., $v_{\mathrm{GS}}=0 \mathrm{~V}$. High-voltage $\mathrm{Si}, \mathrm{Si}-\mathrm{SJ}, \mathrm{SiC}$ and $\mathrm{GaN}$ devices are tested up to a $V_{\mathrm{m}}$ of $400 \mathrm{~V}$. To make a comparative study, twelve FETs are selected such that they have a current rating around $30 \mathrm{~A}$. The part numbers, device technologies and other important details from the datasheets of the selected FETs are tabulated in Table I.

\section{A. Experimental Setup and the Measurement Process}

$Q V$ patterns related to device output capacitances are experimentally obtained using the Sawyer-Tower technique, which relies on only two voltage measurements [9], [21]. ${ }^{1}$ The circuit consists of a signal generator, a high-voltage amplifier, a fixed linear capacitor known as the reference capacitor, $C_{\text {ref }}$, and the device under test (DUT), as shown in Fig. 2. Since $v_{\mathrm{GS}}=$ $0 \mathrm{~V}$, the DUT is essentially a capacitance equal to $C_{\mathrm{o}}$ for positive $v_{\mathrm{DS}}$ values [9]. The series combination of the DUT and $C_{\text {ref }}$ is subjected to a large-signal input voltage $v_{\text {IN }}$ created by amplifying a low-voltage signal $v_{\mathrm{S}}$, having an excitation frequency of $f$. In steady state, a dc bias $\left(V_{\mathrm{REF}}\right)$ is built across $C_{\text {ref }}$; this renders $v_{\mathrm{DS}}$ to vary between $0 \mathrm{~V}$ and $V_{\mathrm{m}}$ [9]. Since the same current flows through $C_{\mathrm{o}}$ and $C_{\text {ref }}$ in steady state, the ac voltage $\left(v_{\text {ref }}=v_{\mathrm{REF}}-V_{\mathrm{REF}}\right)$ across $C_{\text {ref }}$ is proportional to the variation of charge in both $C_{\text {ref }}$ and $C_{\mathrm{o}}$ [9]: i.e.,

$$
Q_{\mathrm{o}} \propto C_{\mathrm{ref}} \cdot v_{\mathrm{ref}} .
$$

\footnotetext{
${ }^{1}$ The two voltages $v_{\mathrm{REF}}$ and $v_{\mathrm{IN}}$ are measured with a Tektronix MDO3104 oscilloscope $(1 \mathrm{GHz})$ accompanied by TPP1000 passive voltage probes (1 $\mathrm{GHz}$ ). The probe-end uses the MMCX square-pin adapter 131-9717-xx.
}

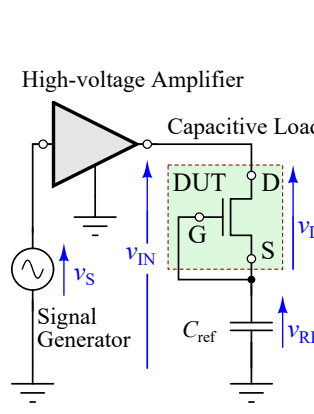

(a)

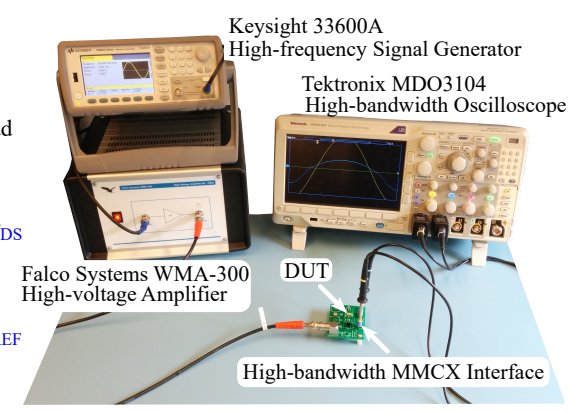

(b)
Fig. 2. (a) Schematic and (b) the experimental test setup of the Sawyer-Tower measurement technique used to analyse large-signal output capacitance.

Finally, the $Q V$ curves are extracted from the measured $v_{\mathrm{REF}}$ and $v_{\mathrm{IN}}$ data by using a simple MATLAB ${ }^{\circledR}$ script. $^{2}$

Fig. 3 plots experimental $Q V$ curves of the selected devices for $\quad=400 \mathrm{~V}$ and $\quad 100 \mathrm{kHz}$. The resulting $E_{\text {diss }}$ is calculated by taking the area (shaded in orange in each sub figure) between the charging (solid red line) and discharging (solid blue line) paths. As the peak output voltage of the highvoltage amplifier was limited to $\pm 150 \mathrm{~V}$ (this limits $V_{\mathrm{m}}$ to 300 $\mathrm{V})$, a HF transformer was utilized at the output of the amplifier to boost the voltage, so that $V_{\mathrm{m}}=400 \mathrm{~V}$ was achievable. A $C_{\text {ref }}$ of $1 \mathrm{nF}$ was employed for all the devices, except for the $\mathrm{Si}$ devices $2-4$, where a $C_{\text {ref }}$ of $47 \mathrm{nF}$ was used. This is because the large $Q_{\mathrm{o}}$ values $(\geq 250 \mathrm{nC}$ ) of these three devices cause too large a variation in $v_{\text {ref }}$ for $C_{\text {ref }}=1 \mathrm{nF}$, and hence a drop in the available $v_{\text {DS }}$ swing. [9].

\section{B. Comparison of QV Patterns at $400 \mathrm{~V}$ and $100 \mathrm{kHz}$}

Fig. 3 shows that the DUTs exhibit diverse $Q V$ patterns, even within the same semiconductor types. In the Si family, the planar-Si structure shows negligible hysteresis while the SJ counterparts exhibit significant hysteresis with $E_{\text {diss }}$ values greater than $1 \mu \mathrm{J}$. The $\mathrm{SJ}$ devices show a distinct knee-type behaviour in their discharging paths (indicated by a green circle), around where the region corresponding to $E_{\text {diss }}$ is much larger, while the respective charging paths show much smoother transitions. The hysteretic area ceases around 200 $\mathrm{V}$, beyond which the two paths coincide. SJ devices show much larger $Q_{\mathrm{o}}$ values ( $\geq 250 \mathrm{nC}$ ), while other devices-with comparable or lower values of on-state resistance, $R_{\mathrm{DS}(\mathrm{on})}-$ show much lower $Q_{\mathrm{o}}$ values $(\leq 100 \mathrm{nC})$ at $400 \mathrm{~V}$.

The WBG devices SiC-1 and GaN-1 hardly show any hysteresis. The barely visible area between the charge-discharge curves of these two devices and the devices $\mathrm{Si}-1$ and $\mathrm{SiC}-3$, is symmetrically distributed within the whole $v_{\text {DS }}$ range (i.e., the widening in the hysteretic area is symmetrical about $\approx V_{\mathrm{m}} / 2$ ). An interesting observation is that the patterns of the cascode structures of the WBG devices (SiC-4 and GaN-4) deviate from their non-cascode counterparts, showing the knee-type behaviour characteristic to SJ structures. Furthermore, the

\footnotetext{
${ }^{2}$ More details on the Sawyer-Tower measurement technique can be found in the works by Perera et al. [9] and Zulauf et al. [2]
} 
TABLE I

Devices Evaluated in the Study

\begin{tabular}{|c|c|c|c|c|c|c|c|}
\hline Index & Technology & Voltage (V) & Part Number & Manufacturer & $\begin{array}{l}\text { Current Rating (A) } \\
@ T_{\mathrm{C}}=25{ }^{\circ} \mathrm{C}\end{array}$ & $\begin{array}{l}R_{\mathrm{DS}(\text { on) }}(\mathrm{m} \Omega) \\
\text { typical }\end{array}$ & Package \\
\hline $\mathrm{Si}-1$ & $\mathrm{Si}$ (planar) & 500 & SiHG32N50D & Vishay Siliconix & 30 & 125 & TO-247 \\
\hline $\mathrm{Si}-2$ & $\mathrm{Si}-\mathrm{SJ}$ & 650 & NTHL110N65S3F & ON Semiconductor & 30 & 98 & TO-247 \\
\hline $\mathrm{Si}-3$ & $\mathrm{Si}-\mathrm{SJ}$ & 650 & IPW65R110CFD & Infineon & 31 & 99 & TO-247 \\
\hline $\mathrm{Si}-4$ & $\mathrm{Si}-\mathrm{SJ}$ & 650 & STW38N65M5 & STMicroelectronics & 30 & 73 & TO-247 \\
\hline $\mathrm{SiC}-1$ & $\mathrm{SiC}$ & 700 & MSC090SMA070S & Microsemi & 25 & 90 & D3PAK \\
\hline $\mathrm{SiC}-2$ & $\mathrm{SiC}$ & 650 & SCT3080AL & ROHM Semiconductor & 30 & 80 & TO-247 \\
\hline $\mathrm{SiC}-3$ & $\mathrm{SiC}$ & 900 & C3M0065090D & Cree & 36 & 65 & TO-247 \\
\hline $\mathrm{SiC}-4$ & $\mathrm{SiC}$ (cascode) & 650 & UF3C065080K3S & UnitedSiC & 31 & 80 & TO-247 \\
\hline $\mathrm{GaN}-1$ & $\mathrm{GaN}$ & 650 & GS66508T & GaN Systems & 30 & 50 & $\operatorname{GaN} P X$ \\
\hline $\mathrm{GaN}-2$ & $\mathrm{GaN}$ & 600 & IGOT60R070D1 & Infineon & 31 & 55 & PG-DSO-20-87 \\
\hline $\mathrm{GaN}-3$ & $\mathrm{GaN}$ & 600 & PGA26E07BA & Panasonic & 31 & 56 & DFN $8 X 8$ \\
\hline $\mathrm{GaN}-4$ & GaN (cascode) & 650 & TPH3212PS & Transphorm & 27 & 72 & TO-220 \\
\hline
\end{tabular}
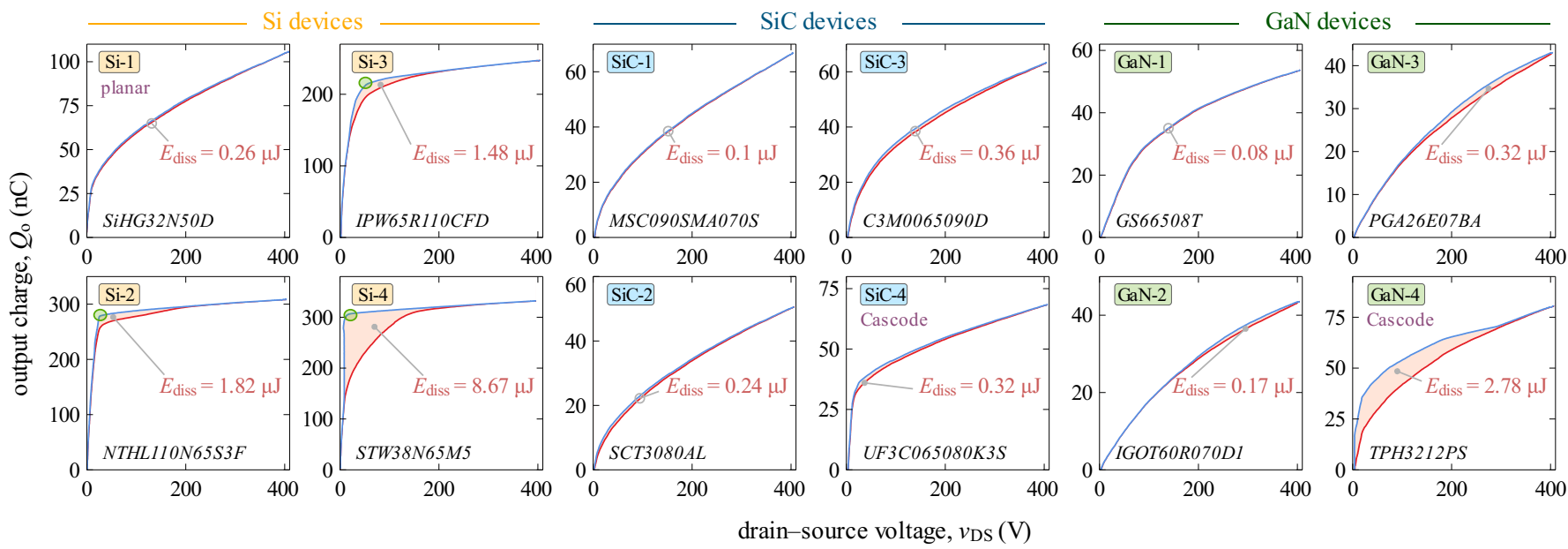

Fig. 3. Experimental $Q V\left(Q_{\mathrm{o}}\right.$ versus $\left.v_{\mathrm{DS}}\right)$ curves of twelve different high-voltage transistors (current rating around $\left.30 \mathrm{~A}\right)$ : planar-Si device $\mathrm{Si}-1, \mathrm{Si}-\mathrm{SJ}$ devices $\mathrm{Si}-2$ to $\mathrm{Si}-4, \mathrm{SiC}$ devices SiC-1 to SiC-4 and GaN devices GaN-1 to GaN-4. The details of the devices are listed in Table I. The solid red and blue lines correspond to charging and discharging paths, respectively; the hysteresis energy loss $E_{\text {diss }}$ is indicated by the area between the two curves (shaded in orange colour). The measurements were performed using the Sawyer-Tower technique at $V_{\mathrm{m}}=400 \mathrm{~V}$. The excitation signal is a sinusoid of $f=100 \mathrm{kHz}$. A $C_{\mathrm{ref}}$ of $1 \mathrm{nF}$ was used for all the measurements, except for the Si devices 2-4, where a $C_{\text {ref }}$ of $47 \mathrm{nF}$ was used.

cascode GaN device shows significant hysteresis compared to other $\mathrm{GaN}$ devices.

These observations are only indicative of the soft-switching performance of these devices at $V_{\mathrm{m}}=400 \mathrm{~V}$ and $f=100$ $\mathrm{kHz}$. A complete understanding requires investigation into their behaviour at different $V_{\mathrm{m}}$ and $f$ values, which is the subject of the next two sections of this paper.

\section{Voltage Dependence of $Q V$ Patterns}

The dependence of $Q V$ patterns on voltage amplitude is investigated in this section. The aim is to identify if the hysteresis patterns emerge only within a certain voltage range, and if so, how they compare with different devices and semiconductor technologies. First, to provide a general view on the selected devices, Fig. 4 compares the variation $E_{\text {diss }}$ with $V_{\mathrm{m}}$ for all the devices. Then, the $Q V$ patterns of each device is individually presented in Fig. 5 to explain their dependence on $V_{\mathrm{m}}$. The excitation frequency is kept fixed at $100 \mathrm{kHz}$.

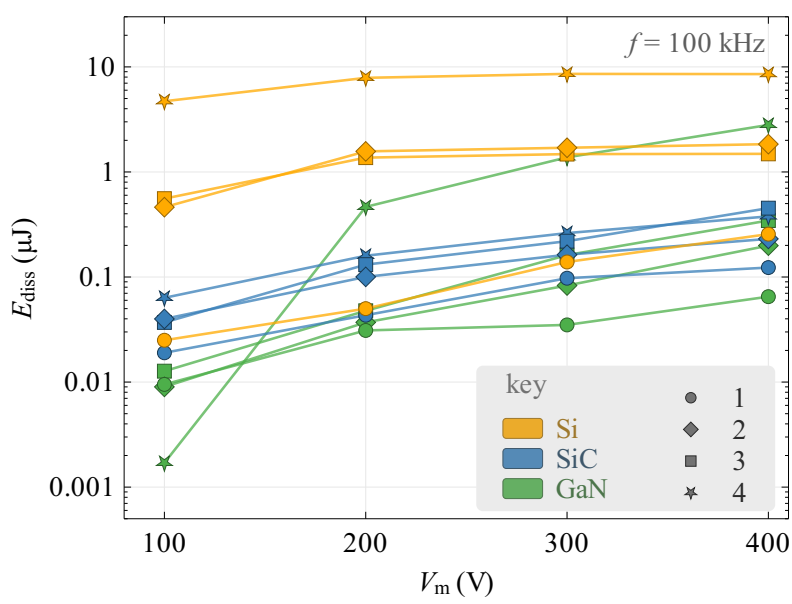

Fig. 4. Variation of $E_{\text {diss }}$ with different $V_{\mathrm{m}}$ values for the twelve tested devices. $V_{\mathrm{m}}$ is varied between 100 and $400 \mathrm{~V}$ at $100 \mathrm{~V}$ steps. The results are obtained with the Sawyer-Tower circuit at $100 \mathrm{kHz}$. 


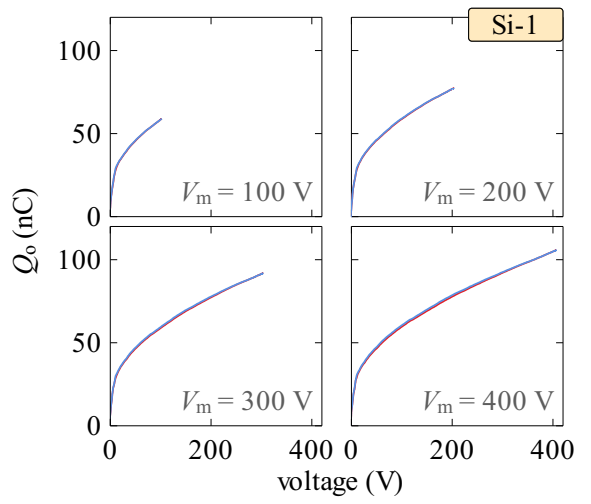

(a)

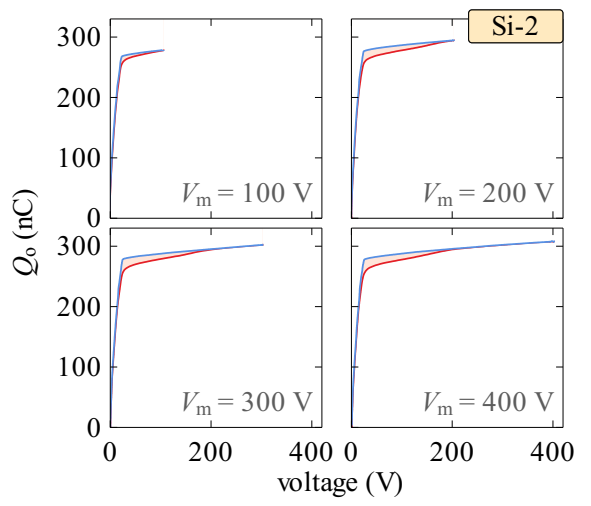

(b)

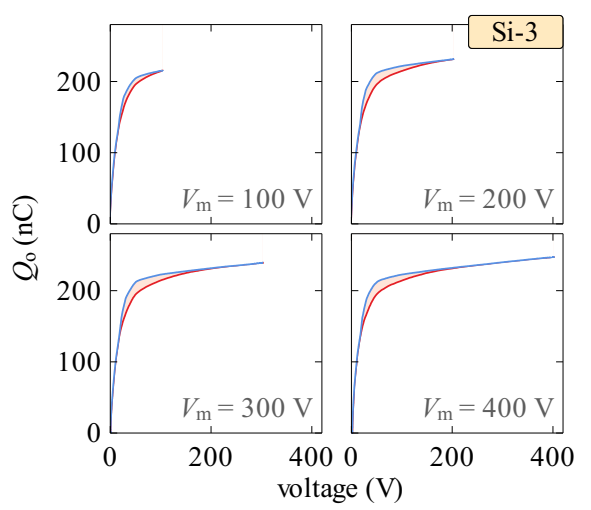

(c)

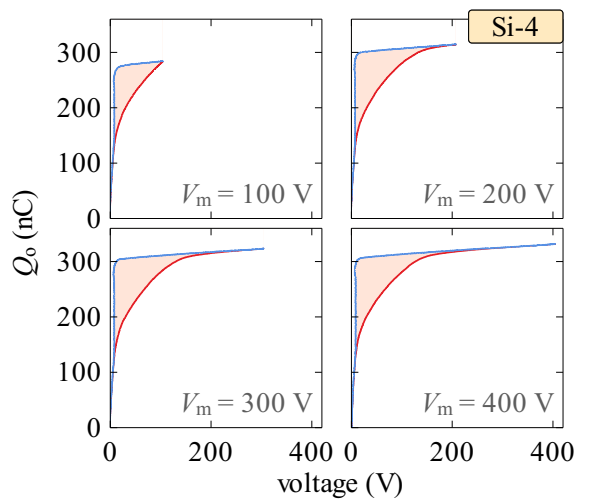

(d)

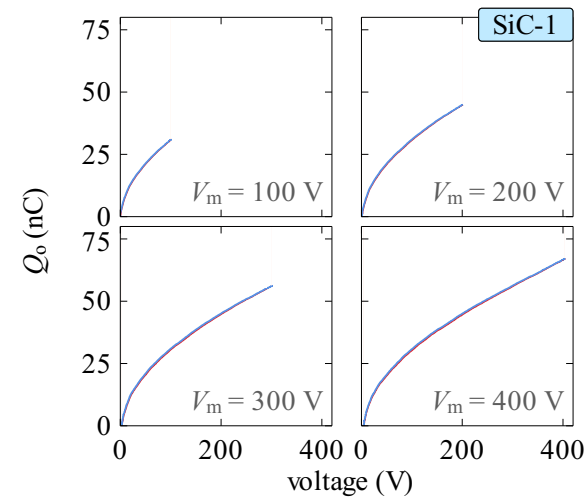

(e)

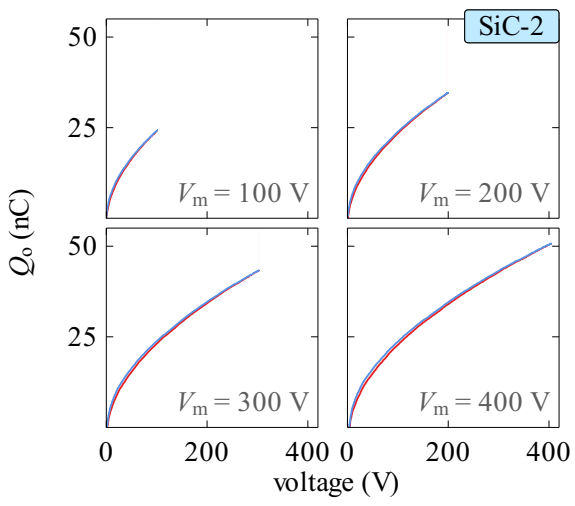

(f)

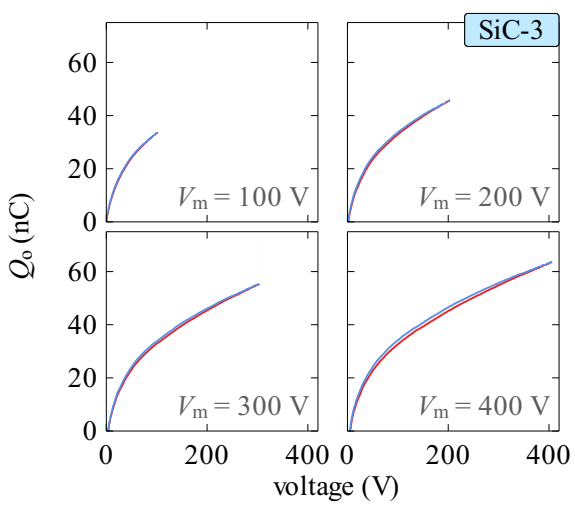

(g)

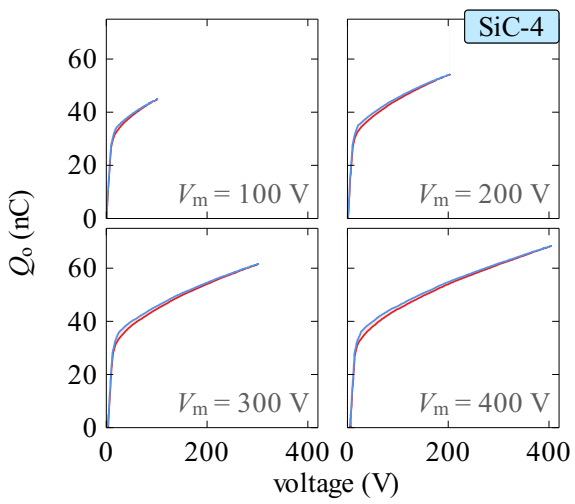

(h)

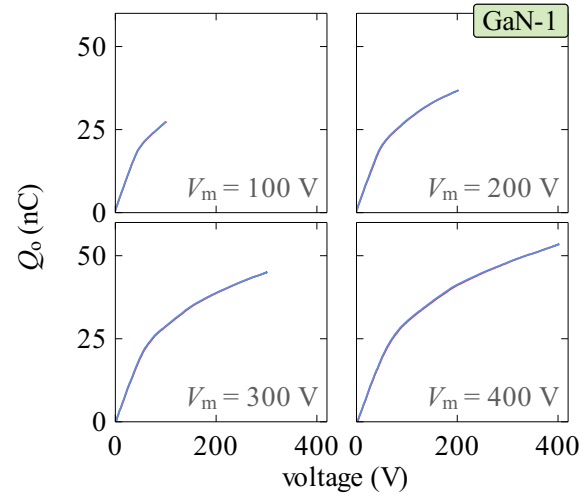

(i)

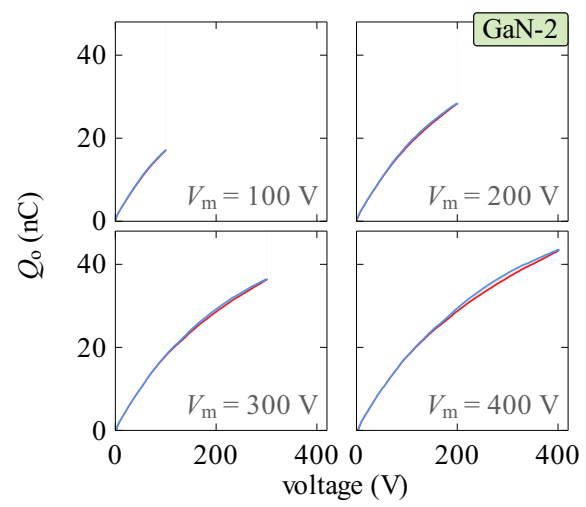

(j)

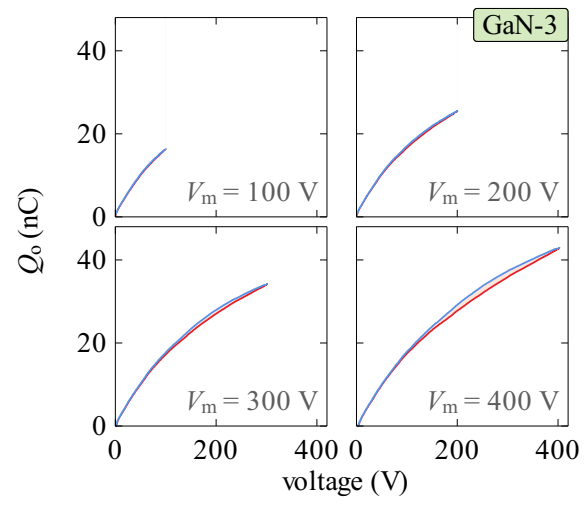

(k)

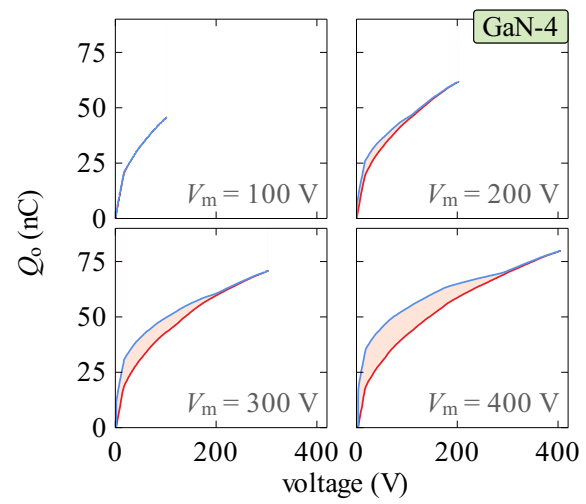

(1)

Fig. 5. Experimental results showing the variation of $Q V$ hysteresis patterns for various $V_{\mathrm{m}}$ values (at $100 \mathrm{kHz}$ ) for all the tested devices. The $Q V$ patterns exhibit diverse dependencies on $V_{\mathrm{m}}$, among different devices and semiconductor technologies. 


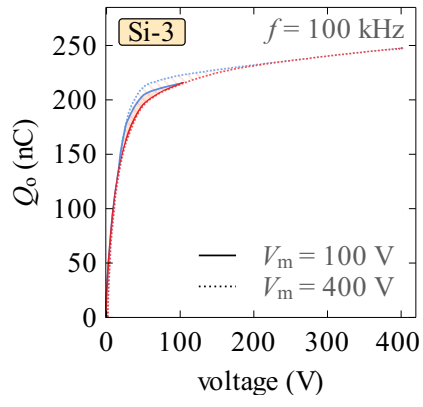

(a)

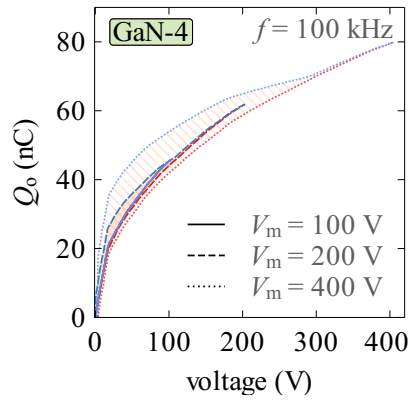

(b)
Fig. 6. Superimposition of $Q V$ hysteresis patterns of the devices (a) Si-3 and (b) GaN-4. The hysteresis pattern of device $\mathrm{Si}-3$ at $400 \mathrm{~V}$ is a clear extension of the pattern at $100 \mathrm{~V}$, with coincidental charging paths at each voltage. Device GaN-4 shows no hysteresis at $100 \mathrm{~V}$; and at 200 and $400 \mathrm{~V}$ it shows hysteresis, but unlike device $\mathrm{Si}-3$, it shows non-coincidental charging paths at each voltage.

A clear observation in Fig. 4 is that the Si-SJ devices show a saturation of their $E_{\text {diss }}$ values as $V_{\mathrm{m}}$ passes $200 \mathrm{~V}$. This can be explained by observing Figs. 5(b) to 5(d). The hysteresis patterns exist even at $100 \mathrm{~V}$ and continue to grow up to 200 V. However, after $200 \mathrm{~V}$, the charging and discharging paths coincide resulting in no hysteretic area. This suggests that the $\mathrm{Si}$-SJ hysteresis is a low-voltage phenomenon, and that operation beyond, for example $200 \mathrm{~V}$ in the studied cases, do not result in additional hysteresis energy losses. This could also be related to their significantly large $C_{\mathrm{o}}$ values (usually $10-100 \mathrm{nF}$ ) in the low $v_{\mathrm{DS}}$ range, which can be up to three orders-of-magnitude larger compared to the values at $400 \mathrm{~V}$.

The devices $\mathrm{Si}-1, \mathrm{SiC}-1$ and $\mathrm{GaN}-1$, as expected from their $400-\mathrm{V}$ results, exhibit no appreciable hysteresis even at low voltages-see Figs. 5(a), 5(e) and 5(i). Only a barelyvisible and symmetrically-spread hysteresis is present. However, Fig. 4 indicates that these devices show, although lower in value, an increasing $E_{\text {diss }}$ with $V_{\mathrm{m}}$. A possible reason for this is discussed in Section V-C.

The importance of the graphical observation of hysteresis patterns becomes apparent by looking into the $\mathrm{GaN}$ devices 1 to 3 , which are from three different manufacturers. According to Fig. 4, the three devices (marked by circle, diamond and square symbols in green) show similar increasing-trends in their $E_{\text {diss }}$ values with $V_{\mathrm{m}}$. However, a major anomaly is observed in devices $\mathrm{GaN}-2$ and $\mathrm{GaN}-3$ when their $Q V$ patterns are looked into-see Figs. 5(j) and 5(k). The hysteresis area of the two devices widens in the high $v_{\text {DS }}$ range (above 200 $\mathrm{V}$ ), unlike in device GaN-1, which shows no such swelling. The hysteresis appears only for cases with $V_{\mathrm{m}} \gtrsim 100 \mathrm{~V}$, and the hysteresis pattern widens with increasing $V_{\mathrm{m}}$. On the other hand, there is no visible hysteresis present at voltages below $100 \mathrm{~V}$ for these two devices ( $V_{\mathrm{m}}$ values of 20 and $50 \mathrm{~V}$ were also tested and results showed no hysteresis); in this case, they act similar to device $\mathrm{GaN}-1$, but in stark contrast to SJ devices, which clearly show a low-voltage hysteresis. This suggests that for these two GaN structures, $C_{\mathrm{o}}$ hysteresis is a phenomenon that occurs only in the high $v_{\mathrm{DS}}$ range. In

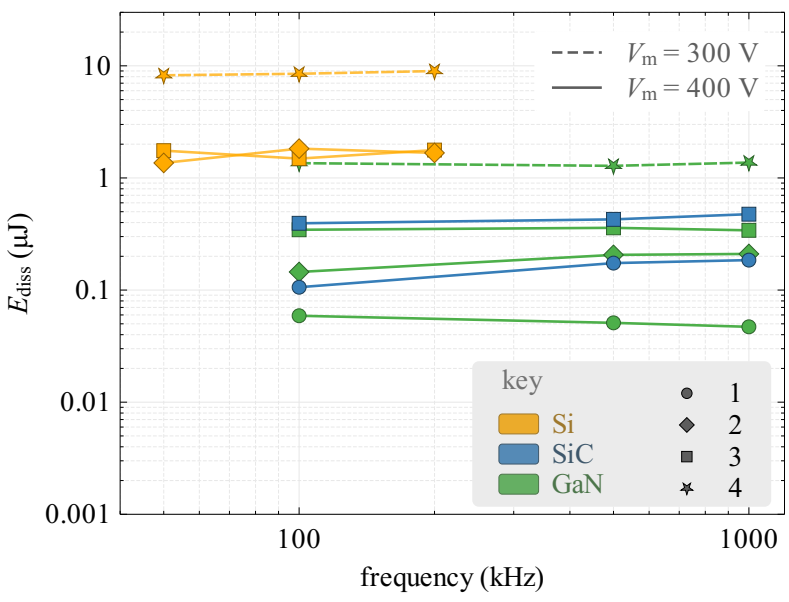

Fig. 7. Variation of $E_{\text {diss }}$ versus $f$ for selected devices. The results are obtained using the Sawyer-Tower circuit with a variable frequency sinusoidal excitation signal. As the Si devices have a large output capacitance value at the low $v_{\text {DS }}$ range, they could only be tested up to $200 \mathrm{kHz}$ due to increased distortion of $v_{\mathrm{IN}}$, reasons of which are explained in the research article by Perera et al. [9].

addition, as Fig. 4 shows, device GaN-3 exhibits much larger $E_{\text {diss }}$ values compared to device $\mathrm{GaN}-2$, which is also verified by the relatively larger hysteresis patterns of the former.

The superimposition of $Q V$ patterns corresponding to different $V_{\mathrm{m}}$ values on the same plot yields additional details between technology-specific differences in hysteresis losses. Fig. 6(a) shows that for device $\mathrm{Si}-3$, the charge paths coincide for 100 - and $400-\mathrm{V}$ cases. However, for the cascode $\mathrm{GaN}$ device, as Fig. 6(b) shows, neither the charging nor the discharging paths show any coincidence for the considered voltages $(100,200$ and $400 \mathrm{~V})$, suggesting a non-uniform dependence of the $Q V$ patterns with $V_{\mathrm{m}}$. The device also shows a large increase of its $E_{\text {diss }}$ value from $100 \mathrm{~V}$ to $200 \mathrm{~V}$ in Fig. 4. This is explained by its $Q V$ hysteresis patterns that appear to take place only if $V_{\mathrm{m}}$ is above $100 \mathrm{~V}$, as Fig. 5(1) indicates. Even above $200 \mathrm{~V}$, the shapes of the hysteresis areas are quite different to other $\mathrm{GaN}$ devices. The shapes are more similar to that of $\mathrm{Si}-\mathrm{SJ}$ devices.

\section{FREQUENCY DEPENDENCE OF $Q V$ PATTERnS}

In this section, the frequency dependence of $E_{\text {diss }}$ is investigated, while keeping the excitation voltage fixed. Fig. 7 plots experimental results for different device structures up to $1 \mathrm{MHz}$. None of the devices exhibit significant dependence on frequency in the considered range. This becomes also apparent by looking at the hysteresis patterns of the devices. Fig. 8(a) shows that the $Q V$ patterns are almost identical for the SJ device $\mathrm{Si}-2$, when excited at three different frequencies $(50,100$ and $200 \mathrm{kHz}$ ), explaining the non-existence of any significant frequency dependence. A similar frequency independence can be seen in the $Q V$ patterns of the WBG device GaN-1, up to $1 \mathrm{MHz}$-see Fig. 8(b). However, some works in the literature show that there is a frequency dependence for certain WBG families, whose effects become prominent only above 5-10 $\mathrm{MHz}$; this aspect is discussed in Section V-B. 


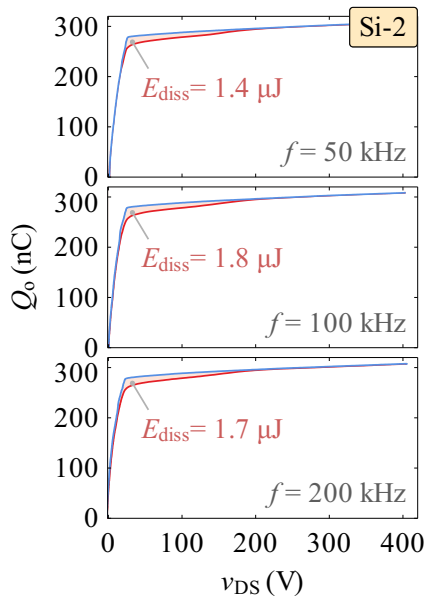

(a)

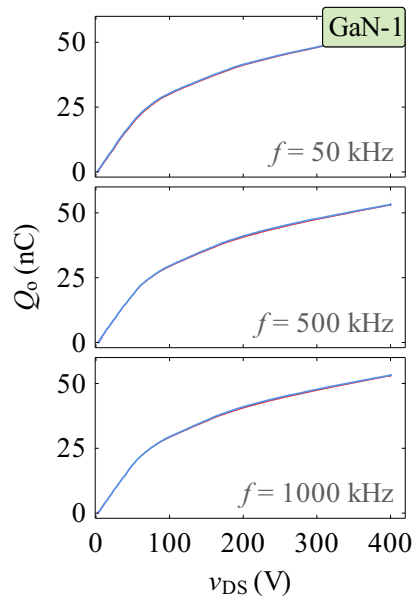

(b)
Fig. 8. (a) For different $f$ values, similar $Q V$ hysteresis patterns are observed in device $\mathrm{Si}$-2. (b) Device GaN-1 shows no hysteresis and maintains a $Q V$ pattern that is independent of $f$ up to $1 \mathrm{MHz}$.

The impact of hysteresis energy loss is important at high frequencies, as the resulting power loss, $P_{\text {diss }}$, scales with $f$ :

$$
P_{\text {diss }}=f \cdot E_{\text {diss }} .
$$

For a given $P_{\text {diss }}$ value, the maximum operating frequency of a certain device is limited by its $E_{\text {diss }}$ value. For instance, at $400 \mathrm{~V}$ and $1 \mathrm{MHz}$, device GaN-4 would result in a power loss greater than $1 \mathrm{~W}$, while device GaN-1 would cause a loss much less than $0.1 \mathrm{~W}$. If a device has a frequency-dependent hysteresis loss, the details of its $E_{\text {diss }}$ as a function of $f$ is required up to the test frequency to estimate $P_{\text {diss. }}$. For such cases, (3) can be modified to show the frequency dependence of $E_{\text {diss }}$ as

$$
P_{\text {diss }}=f \cdot E_{\text {diss }}(f) .
$$

This shows that, if a device has an increasing $E_{\text {diss }}$ with $f$, then it greatly reduces the device's usability, specially at VHF frequencies. However for the considered devices and up to 1 $\mathrm{MHz}$, as Fig. 7 suggests, (3) can be utilized to obtain a good estimate of $P_{\text {diss }}$ by using an $E_{\text {diss }}$ value measured at a much lower frequency, such as $100 \mathrm{kHz}$.

\section{DISCUSSION}

The existing knowledge on the origins of $C_{\mathrm{o}}$ hysteresis losses and the behaviour of these losses beyond $1 \mathrm{MHz}$ are discussed in this section. Finally, a general categorization of $Q V$ hysteresis patterns are presented.

\section{A. The Origins of Output-Capacitance Hysteresis Losses}

Since the report of hysteresis losses observed in Si-SJ FETs [11], several research works have investigated and modelled the origin of these losses with a certain level of experimental validation. A first investigation was presented by Roig et al. for Si-SJ structures [12]. The authors associated the $C_{\mathrm{o}}$ hysteresis to trapped stranded charges $\left(Q_{\mathrm{STR}}\right)$, using mixedmode simulations. They studied both multi-epitaxy multiimplant (MEMI) and trench-filled epitaxial growth (TFEG)
SJ structures, and reported that the former is more prone to $E_{\text {diss }}$ losses. The claims were further studied and confirmed by Zulauf et al. [17]. The more recent work by Lin further studied TFEG structures with numerical simulations and reported that the finite velocities of the carriers in the semiconductor are the root cause for $C_{\mathrm{o}}$ hysteresis in those devices [22].

Some initial studies on the origin of $C_{\mathrm{o}}$ hysteresis losses in GaN-on-Si HEMTs have also been reported [14], [23]. Guacci et al. investigated a $600-\mathrm{V}$ device with a $p$-GaN ohmic gate (device GaN-2 studied in our work) [14]. The study proposed that the cause for the observed hysteresis loss is linked to the non-ideal insulating properties of the GaN carbon-doped buffer, which result in a parasitic resistive behaviour for the drain-substrate capacitance. Such hypothesis was confirmed by the introduction of an improved GaN buffer design, which resulted in a reduced hysteresis loss. Zhuang et al. modelled and analysed a depletion-mode (D-mode) GaN-on-Si device, and consequently, identified additional $C_{\mathrm{o}}$ loss mechanisms [23]: 1) resistive losses in the GaN stack; 2) resistive losses in the Si substrate, related to the doping concentration; and 3) losses in the GaN stack due to traps.

The GaN devices studied in this work, to the best of our knowledge, belong to three different device architectures: device GaN-1 is an e-mode device based on a Schottky $p$ GaN gate structure; devices GaN-2 and GaN-3 are e-mode devices featuring an ohmic $p-\mathrm{GaN}$ gate contact [24]; and $\mathrm{GaN}$ 4 is a cascode device. Interestingly, the $Q V$ patterns observed in Section III also show three different variations for these devices: device $\mathrm{GaN}-1$ shows no hysteresis, devices $\mathrm{GaN}-2$ and GaN-3 show hysteresis only above $100 \mathrm{~V}$, and the cascode structure shows a significantly large hysteresis loss. These observations suggest that additional investigations from device manufactures are required, as complete knowledge on device structures are proprietary.

As for SiC devices, Bura et al. suspected that the trapping in the dielectric interface states could be the reason for related hysteresis losses [15]. The work by Zulauf et al. confirms this suspicion and states that the loss mechanism should be different to GaN-on-Si HEMTs [18]. To the best of our knowledge, a conclusive analysis on the origin of $C_{\mathrm{o}}$ hysteresis in $\mathrm{SiC}$ devices is yet to be published. The lower research interest in this could be due to the fact that $\mathrm{SiC}$ devices are mainly utilized in the $\mathrm{kV}$ range, where the converter switching frequencies are not high, and thus, the limited attention to hysteresis losses.

In summary, the $C_{\mathrm{o}}$ hysteresis losses in $\mathrm{Si}-\mathrm{SJ}$ devices appear to be understood better in comparison to that in WBG devices. On the other hand, it could be argued that, since WBG devices are still emerging, unlike the $\mathrm{Si}$ technology, the original reasons of these losses could also vary, as researchers and manufacturers try new, and if not ambitious, device designs. Nevertheless, it is recommended that the manufacturers acknowledge these losses, with the basic information on quantitative values. The knowledge of these losses (with voltage and frequency) is imperative for the design of high-frequency resonant converters, from the circuit engineer's perspective. 


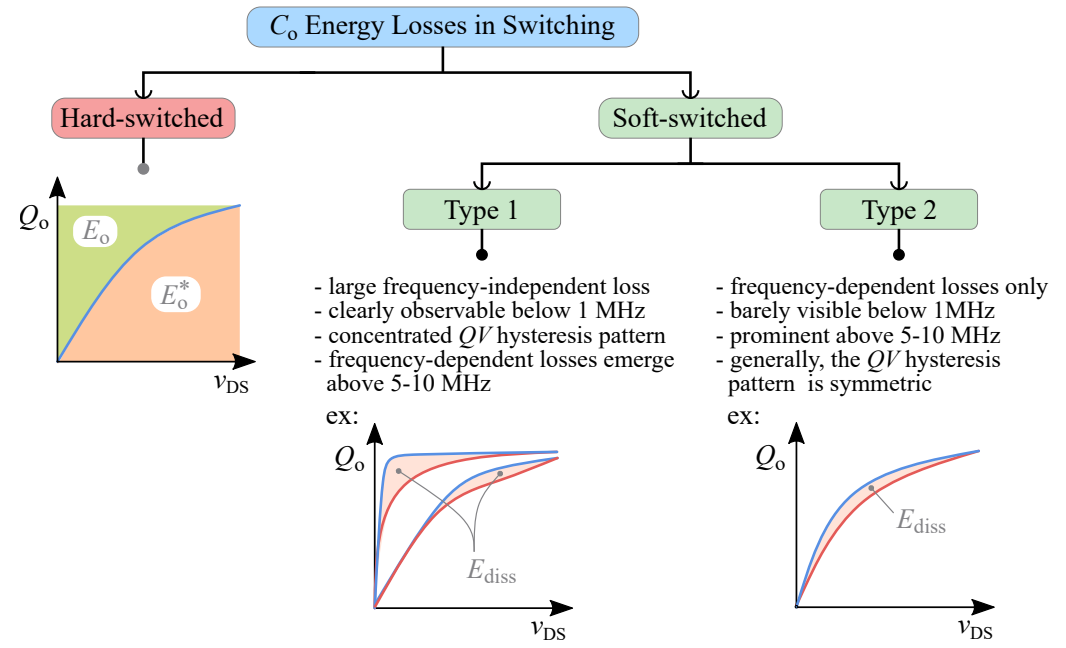

Fig. 9. Categorization of $C_{\mathrm{o}}$-related energy losses in hard-switching and soft-switching power electronic topologies. Energy losses in a soft-switched device can be further categorized into two types based on the shape of its $Q V$ hysteresis pattern. Note: $E_{\mathrm{o}}^{*}$ is the co-energy component related to hard-switching circuits [10].

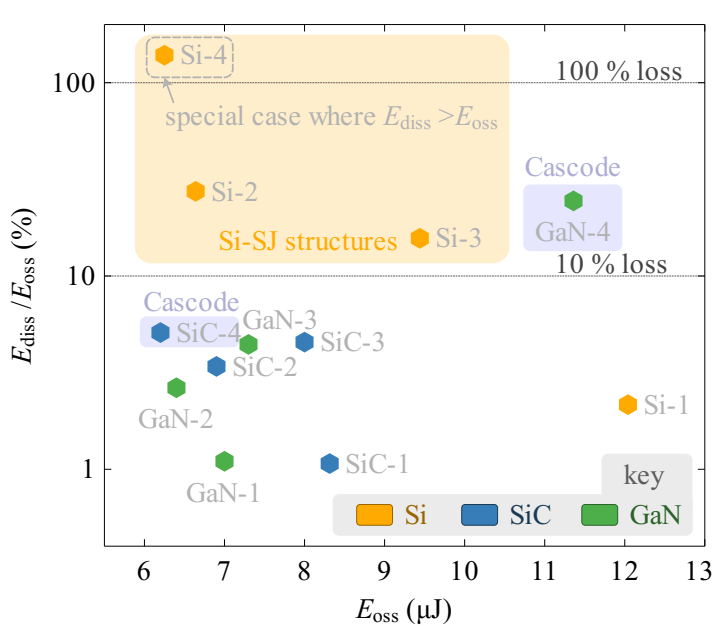

Fig. 10. $E_{\text {diss }}$ values of the devices tested in the study plotted as a percentage loss of their $E_{\text {oss }}$ values. The $E_{\text {diss }}$ values are evaluated at $400 \mathrm{~V}$ and $100 \mathrm{kHz}$, while the $E_{\mathrm{oss}}$ values are estimated using datasheet-provided $C_{\text {oss }}$ curves for $400 \mathrm{~V}$.

\section{B. Output-Capacitance Hysteresis Losses Beyond $1 \mathrm{MHz}$}

Our observations in this work indicate that $E_{\text {diss }}$ show negligible frequency dependence below $1 \mathrm{MHz}$. However, certain recent research shows that there is indeed a frequency dependence, whose effects become prominent only above 5$10 \mathrm{MHz}$ [18]-[20], [25]: for example, the non-cascode GaN devices [2], [25] and certain $\mathrm{SiC}$ families [20], [25].

Moreover, as seen for some $\mathrm{SiC}$ devices in the works by Zulauf et al. [18] and Bura et al. [15], the $Q V$ patterns exhibit symmetrically distributed hysteresis patterns at high frequencies; for SiC-3 device a glimpse of this can even be seen at $100 \mathrm{kHz}$ in Fig. 3. It should also be noted that the $\mathrm{SiC}$ devices tested in the work by Zulauf et al. did not exhibit any appreciable frequency-dependence at high frequencies (up to $35 \mathrm{MHz}$ ). However, for some $\mathrm{SiC}$ devices, frequencydependence has been predicted at high frequencies [20], [25].

Certain Si-SJ devices have reported two hysteresis components: a low-frequency hysteresis component with negligible frequency dependence (agreeing with results in Fig. 7), and a high-frequency component with frequency-dependence [18]. The GaN cascode family (used in our study) could be expected to behave similarly; as 1) our work shows a frequencyindependent, but large, hysteresis loss at low frequency for device $\mathrm{GaN}-4$; and 2) the work by Zulauf et al. shows frequency-dependent hysteresis losses at high frequencies for a device from the same family.

\section{Overview}

The output-capacitance-related energy losses in a switching circuit can be broadly categorized into two main components as Fig. 9 shows: 1) hard-switching losses, and 2) softswitching losses. In hard switching, the energy $\left(E_{0}\right)$ stored in
$C_{\mathrm{o}}$ is lost when the device is turned ON, as shown by the area shaded in green. ${ }^{3}$

In relation to soft-switching, two basic scenarios in $C_{\mathrm{o}}$ hysteresis losses can be identified depending on their frequency dependence: 1) a frequency-independent energy loss, i.e., a form of dc energy loss, which is significant at very low frequencies, such as $10-100 \mathrm{kHz}$; and 2) a frequencydependent energy loss that becomes prominent above 5-10 $\mathrm{MHz}$, but insignificant at low frequencies. Based on our observations and the discussion in Section V-B, commercial FETs can potentially be classified into two generic categories based on $Q V$ hysteresis patterns as shown in Fig. 9.

1) Type 1: devices that show a large frequency-independent energy loss at low frequencies and an additional frequency-dependent energy loss at high frequencies. ex: devices Si-2, Si-3, Si-4, GaN-4

2) Type 2: devices that show only frequency-dependent energy losses. They show very small and quite fixed hysteresis losses at frequencies below $1 \mathrm{MHz}$, but increasing losses above 5-10 MHz.

ex: devices Si-1, SiC-1, GaN-1

Recent research has also shown that the frequency-dependent energy losses in type 2, specially for WBG devices, exhibit a nearly-quadratic variation with $V_{\mathrm{m}}$ [18], [25]. This could explain why the $E_{\text {diss }}$ values of devices $\mathrm{Si}-1, \mathrm{SiC}-1$ and $\mathrm{GaN}$ 1 in Fig. 4 increased with $V_{\mathrm{m}}$.

To provide perspective into the levels of energy that correspond to hard-switching and soft-switching loss mechanisms, Fig. 10 plots $E_{\text {diss }}$ values (at $100 \mathrm{kHz}$ ) normalized by the corresponding small-signal $E_{\mathrm{o}}$ values, $E_{\mathrm{oss}}$ (provided in device datasheets),${ }^{4}$ for all the tested devices. As can be observed,

\footnotetext{
${ }^{3}$ There is another loss component called the co-energy loss in hardswitching circuits [10], which is not the focus of this work.

${ }^{4}$ In device datasheets, small-signal output-capacitance-related parameters are given: capacitance, $C_{\mathrm{oss}} ;$ charge, $Q_{\mathrm{oss}}$; and stored energy, $E_{\mathrm{oss}}$.
} 
$E_{\text {diss }}$ values are generally quite small $(<10 \%)$ in comparison to respective $E_{\text {oss }}$ values. However, the Si-SJ structures and the $\mathrm{GaN}$ cascode show more than $10 \%$ of normalised losses. The $\mathrm{SJ}$ device $\mathrm{Si}-4$ shows an anomaly giving an $E_{\text {diss }}$ value greater than its $E_{\text {oss }}$ value. The devices GaN-1 and and SiC-1 offer the lowest normalised loss for $E_{\text {oss }}$ values up to $9 \mu \mathrm{J}$, suggesting the best soft-switching performance up to 1-5 MHz. Beyond this frequency range, frequency dependence of $E_{\text {diss }}$ should be taken into account.

As a final remark, it should be mentioned that, a component sampling analysis would provide additional details about the hysteresis loss variations in different units of the same product.

\section{CONCLUSION}

We have investigated all four prominent power FET categories (planar-Si, Si-SJ, $\mathrm{SiC}$ and $\mathrm{GaN}$ ) for their outputcapacitance-related $Q V$ hysteresis patterns. A categorical overview of hysteresis patterns of different device structures was presented and their frequency dependence was discussed. We have shown that different structures exhibit different dependencies on excitation voltage levels, resulting in diverse hysteresis patterns. For instance, some structures show no hysteresis below $100 \mathrm{~V}$, whereas for another group, hysteresis is a high-voltage (above 150-200 V) phenomenon. These observations are essential in identifying the underlying physical phenomena in output-capacitance-related losses. The modelling of the voltage dependence of $Q V$ hysteresis patterns in spice simulations is essential for a more realistic analysis of switching transients and losses.

\section{REFERENCES}

[1] M. H. Rashid, Ed., Power Electronics Handbook: Devices, Circuits, and Applications Handbook, 3rd ed. Burlington, MA: Elsevier, 2011.

[2] G. Zulauf, S. Park, W. Liang, K. N. Surakitbovorn, and J. Rivas-Davila, "Coss Losses in $600 \mathrm{~V}$ GaN Power Semiconductors in Soft-Switched, High- and Very-High-Frequency Power Converters," IEEE Trans. Power Electron., vol. 33, no. 12, pp. 10748-10763, Dec. 2018.

[3] W. Zhang, F. Wang, D. J. Costinett, L. M. Tolbert, and B. J. Blalock, "Investigation of Gallium Nitride Devices in High-Frequency LLC Resonant Converters," IEEE Trans. Power Electron., vol. 32, no. 1, pp. 571-583, Jan. 2017.

[4] R. L. Steigerwald, "A comparison of half-bridge resonant converter topologies," IEEE Trans. Power Electron., vol. 3, no. 2, pp. 174-182, Apr. 1988.

[5] N. Sokal and A. Sokal, "Class E-A new class of high-efficiency tuned single-ended switching power amplifiers," IEEE Journal of Solid-State Circuits, vol. 10, no. 3, pp. 168-176, Jun. 1975.

[6] J. M. Rivas, Y. Han, O. Leitermann, A. D. Sagneri, and D. J. Perreault, "A High-Frequency Resonant Inverter Topology With Low-Voltage Stress," IEEE Trans. Power Electron., vol. 23, no. 4, pp. 1759-1771, Jul. 2008.

[7] M. Eron, B. Kim, F. Raab, R. Caverly, and J. Staudinger, "The Head of the Class," IEEE Microwave, vol. 12, no. 7, pp. S16-S33, Dec. 2011.

[8] A. Lidow, J. Strydom, M. de Rooij, and D. Reusch, GaN Transistors for Efficient Power Conversion, 2nd ed. Wiley, Sep. 2014
[9] N. Perera, G. Kampitsis, R. van Erp et al., "Analysis of LargeSignal Output Capacitance of Transistors using Sawyer-Tower Circuit," IEEE J. Emerg. Sel. Topics Power Electron., DOI:10.1109/JESTPE.2020.2992946, 2020.

[10] N. Perera, N. Bolier, E. Figini et al., "Analysis of Output Capacitance Co-Energy and Discharge Losses in Hard-Switched FETs," in International Power Electronics and Motion Control Conference (IPEMC2020ECCE Asia), 2020, in press.

[11] J. B. Fedison, M. Fornage, M. J. Harrison, and D. R. Zimmanck, "Coss related energy loss in power MOSFETs used in zero-voltage-switched applications," in 2014 IEEE Applied Power Electronics Conference and Exposition - APEC 2014, Mar. 2014, pp. 150-156.

[12] J. Roig and F. Bauwens, "Origin of Anomalous Coss Hysteresis in Resonant Converters With Superjunction FETs," IEEE Trans. Electron Devices, vol. 62, no. 9, pp. 3092-3094, Sep. 2015.

[13] J. B. Fedison and M. J. Harrison, "Coss hysteresis in advanced superjunction MOSFETs," in 2016 IEEE Applied Power Electronics Conference and Exposition (APEC), Mar. 2016, pp. 247-252.

[14] M. Guacci, M. Heller, D. Neumayr et al., "On the Origin of the CossLosses in Soft-Switching GaN-on-Si Power HEMTs," IEEE J. Emerg. Sel. Topics Power Electron., vol. 7, no. 2, pp. 679-694, Jun. 2019.

[15] D. Bura, T. Plum, J. Baringhaus, and R. W. D. Doncker, "Hysteresis Losses in the Output Capacitance of Wide Bandgap and Superjunction Transistors," in 2018 20th European Conference on Power Electronics and Applications (EPE'18 ECCE Europe), Sep. 2018, pp. P.1-P.9.

[16] A. Raciti, S. A. Rizzo, N. Salerno et al., "Modeling the Hysteresis Power Losses of the Output Parasitic Capacitance in Super Junction MOSFETs," in 2018 International Symposium on Power Electronics, Electrical Drives, Automation and Motion (SPEEDAM), Jun. 2018, pp. $527-532$.

[17] G. D. Zulauf, J. Roig-Guitart, J. D. Plummer, and J. M. Rivas-Davila, "Coss Measurements for Superjunction MOSFETs: Limitations and Opportunities," IEEE Trans. Electron Devices, vol. 66, no. 1, pp. 578584, Jan. 2019.

[18] G. Zulauf, Z. Tong, J. D. Plummer, and J. M. Rivas-Davila, "Active Power Device Selection in High- and Very-High-Frequency Power Converters," IEEE Trans. Power Electron., vol. 34, no. 7, pp. 68186833, Jul. 2019.

[19] N. Perera, M. S. Nikoo, A. Jafari, L. Nela, and E. Matioli, "Coss Loss Tangent of Field-Effect Transistors: Generalizing High-Frequency SoftSwitching Losses," IEEE Trans. Power Electron., vol. 35, no. 12, pp. 12 585-12 589, Dec. 2020.

[20] A. Jafari, M. S. Nikoo, N. Perera et al., "Comparison of Wide-BandGap Technologies for Soft-Switching Losses at High Frequencies," IEEE Trans. Power Electron., vol. 35, no. 12, pp. 12 595-12 600, Dec. 2020.

[21] C. B. Sawyer and C. H. Tower, "Rochelle Salt as a Dielectric," Phys. Rev., vol. 35, no. 3, pp. 269-273, Feb. 1930.

[22] Z. Lin, "Study on the Intrinsic Origin of Output Capacitor Hysteresis in Advanced Superjunction MOSFETs," IEEE Electron Device Letters, vol. 40, no. 8, pp. 1297-1300, Aug. 2019.

[23] J. Zhuang, G. Zulauf, J. Roig, J. D. Plummer, and J. Rivas-Davila, "An Investigation into the Causes of Coss Losses in GaN-on-Si HEMTs," in 2019 20th Workshop on Control and Modeling for Power Electronics (COMPEL), Jun. 2019, pp. 1-7.

[24] E. A. Jones, F. F. Wang, and D. Costinett, "Review of Commercial GaN Power Devices and GaN-Based Converter Design Challenges," IEEE J. Emerg. Sel. Topics Power Electron., vol. 4, no. 3, pp. 707-719, Sep. 2016.

[25] M. S. Nikoo, A. Jafari, N. Perera, and E. Matioli, "New Insights on Output Capacitance Losses in Wide-Band-Gap Transistors," IEEE Trans. Power Electron., vol. 35, no. 7, pp. 6663-6667, Jul. 2020. 